ROM2F-96-53

hep-lat/9610008

\title{
A WILSON-MAJORANA REGULARIZATION FOR LATTICE CHIRAL GAUGE THEORIES
}

\author{
GABRIELE TRAVAGLINI \\ Dipartimento di Fisica, Università di Roma "La Sapienza" \\ I.N.F.N. - Sezione di Roma II, \\ Via della Ricerca Scientifica, 00133 Roma, ITALY
}

\begin{abstract}
We discuss the regularization of chiral gauge theories on the lattice introducing only physical degrees of freedom. This is obtained by writing the Wilson term in a Majorana form, at the expense of the $U(1)$ symmetry related to fermion number conservation. The idea of restoring chiral invariance in the continuum by introducing a properly chosen set of counterterms to be added to the tree level action is checked against one-loop perturbative calculations.
\end{abstract}

Nuclear Physics B (in press) 


\section{Introduction}

The problem of finding a nonperturbative formulation of a chiral gauge theory is still an active field of investigation [1, 2, 3, 4, 5, 6, 7, 8, 9, 10, 11, 12, 13, 14. The root of this difficulty lies in the impossibility of finding a regularization procedure which preserves the chiral symmetry of the classical action functional. QED gives us a first example of this conflict with the Adler-BellJackiw anomaly [15, 16], which spoils the conservation of the axial current. The anomaly term happily accounts for the measured $\pi^{0} \rightarrow 2 \gamma$ decay rate, which otherwise would be suppressed according to the Sutherland-Veltman theorem [17]. In supersymmetric gauge theories a number of correlators identically vanishing in perturbation theory are in fact nonzero thanks to the existence of topologically nontrivial gauge field configurations with important consequences, as spontaneous supersymmetry breaking in certain chiral theories [18, 19, 20]. But even more striking is what happens in the Weinberg-Salam model, because of the severe physical constraints imposed by the cancellation of quark against lepton anomalies, in order to ensure gauge invariance of the theory, and hence renormalizability 21].

The key point is that it is impossible to quantize chiral fermions coupled to gauge fields in a way that simultaneously preserves the gauge symmetry and the validity of the usual axioms of Quantum Field Theory. In the context of the lattice regularization of a chiral gauge theory with gauge group $S U(2)$, we shall study the possibility of restoring chiral symmetry when the cutoff $\Lambda$ (the inverse lattice spacing, $a^{-1}$ ) goes to infinity. In our formulation, discussed in section 2, we shall not need to introduce any unphysical degrees of freedom, as suggested in [3, 22, 23]. In section 3, we will compare the symmetries of the classical action with those of the regularized one. In particular, in section 4 , we briefly discuss on the anomaly structure of the global $U(1)$ currents and on the related question of fermion number nonconservation. Following the 
approach of [1], we shall replace from the start the asymmetrical regularized action $S$ with an (asymmetrical) functional $S+S_{\text {c.t. }}$, where $S_{\text {c.t. }}$ contains all the interactions which are not invariant under the symmetries broken by the regularization (but of course still invariant under the preserved ones). We shall choose these interactions in such a way that their canonical dimension is less than or equal to four, to keep renormalizability. Moving from the observation that the formal continuum theory is BRST-invariant, we shall try to satisfy the related Slavnov-Taylor identities in the regularized theory with action $S+S_{\text {c.t. }}$ in the limit of infinite cutoff, that is up to terms vanishing when $a \rightarrow 0$; this way we fix the coefficients of the interactions in $S_{\text {c.t. }}$. The program we have just outlined is checked in section 5 by performing a few one-loop computations. In the last section we finally draw some conclusions.

\section{Description of the Model}

In this model we shall consider a fermionic undotted Weyl isodoublet! $\chi_{A}^{\alpha}(n)$, $A=1,2$, defined on lattice sites which transforms according to the fundamental representation of the group $S U(2), \chi^{\alpha}(n) \longrightarrow \Omega(n) \chi^{\alpha}(n), \Omega(n) \in S U(2)$. Its dotted partner $\bar{\chi}_{\dot{\alpha}, A}(n)$ transforms as $\bar{\chi}_{\dot{\alpha}}(n) \longrightarrow \bar{\chi}_{\dot{\alpha}}(n) \Omega^{\dagger}(n)$, and is an independent variable from the point of view of (Euclidean) path integration. We focus our analysis on a theory based on the simple gauge group $S U(2)$, whose representations are real, because we want to keep the calculations as simple as possible; i.e. we avoid the introduction of more fermion matter fields, which would otherwise be necessary to cancel local anomalies, as in the Standard Model. This restriction is merely of a technical nature. Of course one can consider different theories with fermions making up anomaly-free representations; the reader can find a discussion concerning the case of the

\footnotetext{
${ }^{1}$ We are using van der Waerden's notation for Weyl spinors [24].
} 
group $S U(5)$ with one generation of $\mathbf{5}^{*}+\mathbf{1 0}$ Weyl fermions in [22]. It is well known that an $S U(2)$ gauge theory with one Weyl fermion in the fundamental representation is plagued by a global anomaly [25]; nevertheless this will not affect our calculations, because they are performed around the trivial vacuum configuration.

Let then $U_{\mu}(n)=\exp \left[\operatorname{iag} A_{\mu}(n+\mu / 2)\right]$ be a link variable belonging to $S U(2)$ 己 and transforming as $U_{\mu}(n) \longrightarrow \Omega(n) U_{\mu}(n) \Omega^{\dagger}(n+\mu)$ under the gauge group. The action functional $S_{T}+S_{Y M}$, where

$$
\begin{aligned}
& S_{T}=\frac{1}{2 a} \sum_{n, \mu} a^{4}\left[\bar{\chi}^{\dot{\alpha}}(n) \bar{\sigma}_{\dot{\alpha} \beta, \mu} U_{\mu}(n) \chi^{\beta}(n+\mu)-\bar{\chi}^{\dot{\alpha}}(n+\mu) U_{\mu}^{\dagger}(n) \bar{\sigma}_{\dot{\alpha} \beta, \mu} \chi^{\beta}(n)\right], \\
& S_{Y M}=-\frac{1}{2 g^{2}} \sum_{n, \mu, \nu} \operatorname{Tr}\left[U_{\mu}(n) U_{\nu}(n+\mu) U_{\mu}^{\dagger}(n+\nu) U_{\nu}^{\dagger}(n)-1\right]+\text { h.c. , }
\end{aligned}
$$

is then invariant with respect to local $S U(2)$ transformations.']

Unfortunately, the theory defined by $S_{T}+S_{Y M}$ does not correctly reproduce the continuum limit of a chiral gauge theory, due to the well-known fermion species doubling. The strategy proposed by Wilson, to circumvent this problem, consists in adding to the action a term chosen in such a way that only one fermion species survives in the continuum limit, $a \rightarrow 0$, having given the spurious states a mass of order $a^{-1}$ [26]. Using Weyl spinors it is possible to choose this term in a way in which only left-handed fermions are involved, that is without introducing any fictitious degree of freedom [3], 22, 23]. This interaction (that we call the Wilson-Majorana term) is

$$
\begin{aligned}
S_{W}= & -\frac{r}{a} \sum_{n, \mu} a^{4}\left\{\bar{\chi}^{\dot{\alpha}}(n)\left(\bar{\chi}^{\dot{\beta}}(n+\mu)-\bar{\chi}^{\dot{\beta}}(n)\right) \epsilon_{\dot{\alpha} \dot{\beta}}+\right. \\
& \left.\left(\chi^{\alpha}(n+\mu)-\chi^{\alpha}(n)\right) \chi^{\beta}(n) \epsilon_{\beta \alpha}\right\},
\end{aligned}
$$

\footnotetext{
${ }^{2}$ Our gauge connection is hermitian and it is defined as $A_{\mu} \equiv A_{\mu}^{a} \sigma^{a} / 2$, where the $\sigma^{a}$ are the usual Pauli matrices.

${ }^{3} \mathrm{~A}$ brief excursus on $\bar{\sigma}$ matrices can be found in the appendix.
} 
where $0<r \leq 1$, and (2.3) is the discretized version of

$$
-\frac{1}{2}(r a) \int d^{4} x\left(\bar{\chi}^{\dot{\alpha}} \square \bar{\chi}^{\dot{\beta}} \epsilon_{\dot{\alpha} \dot{\beta}}+\chi^{\alpha} \square \chi^{\beta} \epsilon_{\beta \alpha}\right),
$$

which is an irrelevant coupling, formally vanishing when the cutoff is removed. We can immediately check that this term gives a mass of order $r a^{-1}$ to the unwanted fermionic degrees of freedom by looking at the two point Green functions of the free theory,

$$
\begin{gathered}
P_{A B}^{\dot{\alpha} \beta}(n) \equiv\left\langle\chi_{A}^{\beta}(n) \bar{\chi}_{B}^{\dot{\alpha}}(0)\right\rangle=\int_{-\frac{\pi}{a}}^{\frac{\pi}{a}} \frac{d^{4} p}{(2 \pi)^{4}} e^{i p n a} \frac{\left(-i a \sum_{\mu} \bar{\sigma}_{\mu}^{\dot{\alpha} \beta} \sin p_{\mu} a\right)}{\triangle(p a)} \delta_{A B}, \\
P_{A B}^{\alpha \beta}(n) \equiv\left\langle\chi_{A}^{\alpha}(n) \chi_{B}^{\beta}(0)\right\rangle=\int_{-\frac{\pi}{a}}^{\frac{\pi}{a}} \frac{d^{4} p}{(2 \pi)^{4}} e^{i p n a} \frac{a W(p a)}{\triangle(p a)} \epsilon^{\alpha \beta} \delta_{A B} \\
P_{\dot{\alpha} \dot{\beta}, A B}(n) \equiv\left\langle\bar{\chi}_{\dot{\alpha}, A}(n) \bar{\chi}_{\dot{\beta}, B}(0)\right\rangle=\int_{-\frac{\pi}{a}}^{\frac{\pi}{a}} \frac{d^{4} p}{(2 \pi)^{4}} e^{i p n a} \frac{a W(p a)}{\triangle(p a)} \epsilon_{\dot{\beta} \dot{\alpha}} \delta_{A B}
\end{gathered}
$$

where

$$
\triangle(p)=D(p)+W^{2}(p)
$$

with

$$
\begin{gathered}
D(p)=\sum_{\mu} \sin ^{2} p_{\mu}, \\
W(p)=2 r \sum_{\mu}\left(1-\cos p_{\mu}\right) .
\end{gathered}
$$

The appearance of two nondiagonal propagators in (2.6), (2.7) is the natural consequence of the explicit breaking of the $U(1)$ symmetry related to fermion number conservation that our regularization entails. When $r \rightarrow 0^{+}$ this symmetry is restored, the nondiagonal propagators vanish, and we have again $2^{4}$ different fermions.

To sum up, the complete tree level regularized action of the theory is

$$
S=S_{T}+S_{Y M}+S_{g f}+S_{W}
$$


In the last equation $S_{g f}$ is the sum of gauge-fixing and ghost terms, and reads, in continuum notation,

$$
S_{g f}=\int d^{4} x\left[\frac{1}{2 \alpha}\left(\partial_{\mu} A_{\mu}^{a}\right)\left(\partial_{\nu} A_{\nu}^{a}\right)+\bar{c}^{a} \partial_{\mu}\left(D_{\mu} c\right)^{a}\right]
$$

where $D_{\mu}^{a b}$ is the covariant derivative in the adjoint representation,

$$
D_{\mu}^{a b}=\partial_{\mu} \delta^{a b}+g \epsilon^{a b c} A_{\mu}^{c} \quad a, b=1,2,3 .
$$

In [27, 28], it has been pointed out that in a chiral gauge theory the nonsymmetrical (e.g. dimensional) regularization requires the introduction of nongauge-invariant counterterms in the ghost sector, making it unavoidable to fix the gauge even on the lattice, in contrast to the case of a theory with vector-like symmetry (where the invariant formulation and the compactness of the group make the functional integral well-defined, without the need of introducing any supplementary condition).

Note that we could also add a Majorana mass term to $S$,

$$
\frac{m}{2} \sum_{n} a^{4}\left[\bar{\chi}^{\dot{\alpha}}(n) \bar{\chi}^{\dot{\beta}}(n) \epsilon_{\dot{\alpha} \dot{\beta}}+\chi^{\alpha}(n) \chi^{\beta}(n) \epsilon_{\beta \alpha}\right],
$$

with the sole effect of redefining the function in (2.10), $W(p a) \longrightarrow W(p a)+m a$. We shall soon see that renormalization generates such a mass term.

\section{Symmetry Properties of the Regularized Action}

Due to the presence of a gauge-fixing term, the action functional $S-S_{W}=$ $S_{T}+S_{Y M}+S_{g f}$ no longer possesses a gauge symmetry. Conversely, it exhibits a BRST invariance [29, 30], summarized by the infinitesimal transformations

$$
\delta \bar{c}^{a}=\bar{\epsilon} / \alpha g\left(\partial_{\mu} A_{\mu}^{a}\right) \quad, \quad \delta c^{a}=-\bar{\epsilon} / 2 \epsilon^{a b l} c^{b} c^{l}
$$




$$
\begin{gathered}
\delta A_{\mu}^{a}=-\frac{\bar{\epsilon}}{g}\left(D_{\mu} c\right)^{a}, \\
\delta \chi=i \bar{\epsilon} c^{a} T^{a} \chi, \quad \delta \bar{\chi}=i \bar{\epsilon} \bar{\chi} T^{a} c^{a},
\end{gathered}
$$

where $\bar{\epsilon}$ is a Grassmann number, and $T^{a}=\sigma^{a} / 2$. However, $S$ is not invariant under the transformations (3.1): once we introduce the Wilson-Majorana term, BRST invariance is lost, as well as the invariance under global $S U(2)$ and $U(1)$ transformations: in the language of a Wilson-like lattice regularization, this is the way anomalies come into play. The breaking of BRST invariance in our model is not a consequence of the form in which we wrote the Wilson term, as stated by the Nielsen-Ninomiya theorem: whatever strategy we use to remove fermion doublers, chiral invariance is lost, if we want to preserve the locality of the theory [31, 32].

Let us now move on to the residual symmetry properties of the model. This question is particularly important here because of the more "asymmetrical" form of our regularization (with respect to the Wilson-Dirac one employed in [1]). This way, we shall still be able to put some restrictions on the possible form of the regularized (and unrenormalized) Green functions $G_{\Lambda}\left(x_{1}, \ldots, x_{n}\right)$, thus explaining some of the results of perturbation theory that could otherwise seem fortuitous. We find that two simple discrete symmetries and a continuous one survive even for $a \neq 0$. In fact, the following three sets of transformations leave the action of the theory invariant:

1.

$$
\begin{gathered}
\chi \longrightarrow e^{i \varphi T_{f}^{2}} \chi \quad \bar{\chi} \longrightarrow \bar{\chi} e^{-i \varphi T_{f}^{2}}, \\
A \longrightarrow e^{i \varphi T_{f}^{2}} A e^{-i \varphi T_{f}^{2}}, \\
c \longrightarrow e^{i \varphi T_{a d j}^{2}} c \quad \bar{c} \longrightarrow \bar{c} e^{-i \varphi T_{a d j}^{2}},
\end{gathered}
$$

2.

$$
\chi \longrightarrow \sigma^{1} \chi \quad \bar{\chi} \longrightarrow \bar{\chi} \sigma^{1}
$$




$$
\begin{gathered}
A \longrightarrow e^{i \pi T_{f}^{1}} A e^{-i \pi T_{f}^{1}} \\
c \longrightarrow e^{i \pi T_{a d j}^{1} c} \quad \bar{c} \longrightarrow \bar{c} e^{-i \pi T_{a d j}^{1}}
\end{gathered}
$$

3.

$$
\begin{gathered}
\chi \longrightarrow \sigma^{3} \chi \quad \bar{\chi} \longrightarrow \bar{\chi} \sigma^{3}, \\
A \longrightarrow e^{i \pi T_{f}^{3}} A e^{-i \pi T_{f}^{3}}, \\
c \longrightarrow e^{i \pi T_{\text {adj }}^{3}} \quad \bar{c} \longrightarrow \bar{c} e^{-i \pi T_{a d j}^{3}},
\end{gathered}
$$

where $T_{r}^{a}, a=1,2,3$ are the generators of the symmetry in the representation $\mathbf{r}$ (then $T_{f}^{a}=\sigma^{a} / 2$ ), and $\varphi$ is a constant. It should be noticed anyway that much of this is a consequence of the well-known property of the generators of $S U(2)$ in their fundamental representation, $\sigma^{2} \sigma^{a} \sigma^{2}=-\sigma^{a}$ *

As we can see, the first one is a continuous global symmetry, whereas the last two involve discrete transformations of the fields. As a consequence, there will be one conserved Noether current, whose expression is

$$
\begin{aligned}
J_{\mu}(n)= & \frac{1}{2}\left[\bar{\chi}^{\dot{\alpha}}(n) \bar{\sigma}_{\dot{\alpha} \beta, \mu} \frac{\sigma_{2}}{2} \chi^{\beta}(n+\mu)+\bar{\chi}^{\dot{\alpha}}(n+\mu) \bar{\sigma}_{\dot{\alpha} \beta, \mu} \frac{\sigma_{2}}{2} \chi^{\beta}(n)\right] \\
& -r\left[\bar{\chi}^{\dot{\alpha}}(n) \frac{\sigma_{2}}{2} \bar{\chi}^{\dot{\beta}}(n+\mu) \epsilon_{\dot{\alpha} \dot{\beta}}-\chi^{\alpha}(n+\mu) \frac{\sigma_{2}}{2} \chi^{\beta}(n) \epsilon_{\beta \alpha}\right] .
\end{aligned}
$$

Since

$$
\bar{\chi}^{\dot{\alpha}}(n) \frac{\sigma_{2}}{2} \bar{\chi}^{\dot{\beta}}(n) \epsilon_{\dot{\alpha} \dot{\beta}}=\chi^{\alpha}(n) \frac{\sigma_{2}}{2} \chi^{\beta}(n) \epsilon_{\beta \alpha}=0,
$$

the formal continuum limit of $(3.5)$ is

$$
J_{\mu}(x)=\bar{\chi}^{\dot{\alpha}}(x) \bar{\sigma}_{\dot{\alpha} \beta, \mu} \frac{\sigma_{2}}{2} \chi^{\beta}(x)
$$

As a simple example, we now discuss the consequences of these symmetries on the general structure, in color space, of the nondiagonal propagator of $\chi$ 
fields $S_{A B}^{\alpha \beta}(n) \equiv\left\langle\chi_{A}^{\alpha}(n) \chi_{B}^{\beta}(0)\right\rangle$. Using the two discrete transformations (3.3), (3.4), we obtain that

$$
S_{A B}^{\alpha \beta}(n)=\left\langle\left(\sigma^{i} \chi^{\alpha}\right)_{A}(n)\left(\sigma^{i} \chi^{\beta}\right)_{B}(0)\right\rangle \quad i=1,3
$$

that is

$$
S_{11}^{\alpha \beta}(n)=S_{22}^{\alpha \beta}(n), \quad S_{12}^{\alpha \beta}(n)=S_{21}^{\alpha \beta}(n)=0 .
$$

Hence $S_{A B}^{\alpha \beta}(n)=C^{\alpha \beta}(n) \delta_{A B}$, which shows that this propagator is diagonal in color space.

\section{Fermion Number Nonconservation and Anomalies}

Let us comment on the breaking of the $U(1)$ symmetry associated with fermion number conservation. In the previous formulation of lattice chiral gauge theories discussed in [1, 22, 23] one introduces

1. the physical left-handed fermionic degrees of freedom, minimally coupled to the gauge fields (in the Dirac notation of [1] we call them $\psi_{L}$ ), and

2. a set of non-interacting, unphysical right-handed fields, $\psi_{R}$, to be not confused with the right-handed physical fields which could possibly be present.

The kinetic term should then be written as

$$
\begin{aligned}
S_{T}= & \frac{1}{2 a} \sum_{n, \mu} a^{4}\left[\bar{\psi}_{L}(n) \gamma_{\mu} U_{\mu}(n) \psi_{L}(n+\mu)-\bar{\psi}_{L}(n+\mu) U_{\mu}^{\dagger}(n) \gamma_{\mu} \psi_{L}(n)\right]+ \\
& \frac{1}{2 a} \sum_{n, \mu} a^{4}\left[\bar{\psi}_{R}(n) \gamma_{\mu} \psi_{R}(n+\mu)-\bar{\psi}_{R}(n+\mu) \gamma_{\mu} \psi_{L}(n)\right]
\end{aligned}
$$


which is nothing but the lattice transcription of

$$
\int d^{4} x\left[\bar{\psi}_{L} \gamma_{\mu}\left(\partial_{\mu}+i g A_{\mu}\right) \psi_{L}+\bar{\psi}_{R} \gamma_{\mu} \partial_{\mu} \psi_{R} \cdot\right]
$$

The $\psi_{R}$ fields allows one to write a Wilson(-Dirac) term transforming, under $U(1)$, like the monomials $\bar{\psi}_{L} \psi_{R}, \bar{\psi}_{R} \psi_{L}$. For example it could be chosen in such a way that its continuum limit is proportional to

$$
a \int d^{4} x\left[\left(\partial_{\mu} \bar{\psi}_{L}\right)\left(\partial_{\mu} \psi_{R}\right)+\left(\partial_{\mu} \bar{\psi}_{R}\right)\left(\partial_{\mu} \psi_{L}\right)\right]
$$

The question of fermion number violation in the context of the theory with a Wilson term written in a Dirac form has been discussed in [3, 22]. In this papers it is argued that fermion number violating correlators can be obtained by applying the cluster decomposition theorem to a Green function like

$$
\left\langle\mathcal{O}_{\Delta F=+2}(x) \mathcal{O}_{\Delta F=-2}(y)\right\rangle
$$

where $\mathcal{O}_{\Delta F=+2}\left(\mathcal{O}_{\Delta F=-2}\right)$ describes a transition in which the variation $\Delta F$ of the fermion number is $+2(-2)$. When $|x-y| \rightarrow \infty$, one gets

$$
\lim _{|x-y| \rightarrow \infty}\left\langle\mathcal{O}_{\Delta F=+2}(x) \mathcal{O}_{\Delta F=-2}(y)\right\rangle=\left\langle\mathcal{O}_{\Delta F=+2}(x)\right\rangle\left\langle\mathcal{O}_{\Delta F=-2}(y)\right\rangle
$$

detailed calculations are then required to check if the r.h.s. of (4.5) is actually nonzero.

Of course, in the Majorana form discussed in this paper, clustering arguments could still be applied. On the other hand, here fermion number nonconservation could arise as a consequence of not having introduced the unphysical right-handed degrees of freedom which would allow us to write a Wilson term à la Dirac. This way we have the interesting possibility of writing non $U(1)$-invariant amplitudes in perturbation theory for finite lattice spacing, without the need of applying clustering arguments to Green functions [3, 22, 23, 14]. Since the Wilson-Majorana term (2.3) breaks fermion 
number conservation, at the lattice level these amplitudes are nonvanishing. Of course non $U(1)$-invariant correlators must vanish in any finite order of perturbation theory when the cutoff is removed; could it happen that, for some nonperturbative field configuration, some of them have a nonzero continuum limit? If such a case occurs, we will immediately be able to identify physical processes in which fermion number is not conserved [33, 34]. There is however some problem in this approach. To begin with, one should require that lattice regularizations reproduce in the $a \rightarrow 0$ limit the Atiyah-Singer index theorem. It requires that, if $n_{L}\left(n_{R}\right)$ is the number of left-handed (right-handed) zero-modes of the Dirac operator in the representation $\mathbf{r}$ of the gauge group $G$ in the external field of a gauge connection $A$ of winding number $k[A]$, then [35, 36, 37, 38]

$$
n_{L}-n_{R}=2 C(\mathbf{r}) k[A]
$$

here $C(\mathbf{r})$ is the Dynkin index of the representation $\mathbf{r}$. Moreover, if $A$ is (anti)self-dual $n_{R}=0\left(n_{L}=0\right)$, so that

$$
\begin{aligned}
& n_{L}=2 C(\mathbf{r}) k[A], \quad n_{R}=0, \quad \text { if } F[A]=\tilde{F}[A], \\
& n_{R}=-2 C(\mathbf{r}) k[A], \quad n_{L}=0, \quad \text { if } F[A]=-\tilde{F}[A] .
\end{aligned}
$$

The index of the Weyl operator in the representation $\mathbf{r}$ in a gauge field of positive winding number $k[A]$ is then given by $2 C(\mathbf{r}) k[A]$. However, when the number of fermions per unit volume is keep finite and the lattice regularized version of the Weyl operator is a square matrix, the index cannot be nonzero, so that the index theorem seems to be not correctly reproduced [39, 40].

An alternative way to state the problem is the following. The number of fermion zero-modes of the (chiral) Weyl operator is deeply related to the

\footnotetext{
${ }^{4}$ We thank the Referee for discussions on this point.
} 
anomaly of the current associated with fermion number. The continuum anomaly equation for the (classically conserved) $U(1)$ current for the Weyl fermion in the representation $\mathbf{r}$ of $G$ (in our case $G=S U(2)$ and $\mathbf{r}=\mathbf{2}$ ) $J_{\mu}=\bar{\chi} \bar{\sigma}_{\mu} \chi$, reads

$$
\partial_{\mu} J_{\mu}=-\frac{1}{32 \pi^{2}}\left(F_{\mu \nu}^{a} \tilde{F}_{\mu \nu}^{a}\right) 2 C(\mathbf{r}),
$$

(recall that $C(\mathbf{2})=1 / 2$ ). By consequence, a discrete $\mathbb{Z}_{2 C(\mathbf{r})}$ subgroup of the anomalous $U(1)$ is preserved at the quantum level. Since in the present case $C(\mathbf{2})=1 / 2$ no such discrete subgroup should survive. The number of fermion zero-modes is $2 \cdot 1 / 2 \cdot k[A]=k[A]$, so that, when $k[A]=1$, one should find a nonzero vacuum expectation value in the external field of an instanton for a single fermion insertion. However, the regularization here employed, which is encoded in the Wilson-Majorana term (2.3), has a discrete $\mathbb{Z}_{2}$ symmetry (which in this case is also a subgroup of the hypercubical symmetry of the lattice)

$$
\chi \longrightarrow-\chi \quad, \quad \bar{\chi} \longrightarrow-\bar{\chi},
$$

which would imply the absence of fermionic condensates, thus not correctly reproducing instanton physics [33, 34].

This problem is also present when considering the $S U(5)$ gauge theory with one left-handed fermion, $\chi^{i}$, in the antifundamental $\left(5^{*}\right)$, and one lefthanded fermion, $\chi_{[i j]}$, in the 2 -index antisymmetric representation (10), discussed in 22]. There, one has $C(\mathbf{1 0})=3 / 2\left(\right.$ and of course $\left.C\left(\mathbf{5}^{*}\right)=1 / 2\right)$. The continuum currents $J_{\mathbf{5}^{*}}^{\mu}, J_{\mathbf{1 0}}^{\mu}$ are then anomalous,

$$
\begin{gathered}
J_{\mathbf{5}^{*}}^{\mu}=\bar{\chi}_{i} \bar{\sigma}^{\mu} \chi^{i}, \partial_{\mu} J_{\mathbf{5}^{*}}^{\mu}=-\frac{1}{32 \pi^{2}}\left(F_{\mu \nu}^{a} \tilde{F}_{\mu \nu}^{a}\right), \\
J_{\mathbf{1 0}}^{\mu}=\bar{\chi}^{[i j]} \bar{\sigma}^{\mu} \chi_{[i j]}, \partial_{\mu} J_{\mathbf{1 0}}^{\mu}=-3 \cdot \frac{1}{32 \pi^{2}}\left(F_{\mu \nu}^{a} \tilde{F}_{\mu \nu}^{a}\right),
\end{gathered}
$$

\footnotetext{
${ }^{5}$ In the external field of an instanton, the (e.g. continuum) theory has no Lorentz (as well as conformal) invariance. This is because the instanton just breaks this symmetry, which is restored after the integration over the instanton collective coordinates.
} 
as well as the fermion number current

$$
J_{F}^{\mu}=J_{5^{*}}^{\mu}+J_{10}^{\mu},
$$

whose anomaly is given by

$$
\partial_{\mu} J_{F}^{\mu}=-4 \cdot \frac{1}{32 \pi^{2}}\left(F_{\mu \nu}^{a} \tilde{F}_{\mu \nu}^{a}\right) .
$$

From (1.12) it follows that the $U(1)$ group associated with fermion number (non)conservation,

$$
U(1): \chi^{i} \longrightarrow e^{i \alpha} \chi^{i}, \quad \chi_{[i j]} \longrightarrow e^{i \alpha} \chi_{[i j]},
$$

has a discrete $\mathbb{Z}_{4}$ subgroup generated by the transformations (4.13) with $\alpha_{m}=(2 \pi / 4) m, m \in \mathbb{Z}$ which is a symmetry of the full quantum theory, since in this case the action functional $S$ transforms as

$$
S \longrightarrow S+i 4 k \alpha_{m}=S+2 \pi i m \text {. }
$$

The $S U(5)$ Wilson-Majorana term reads, in continuum notation,

$$
S_{W}\left[\mathbf{5}^{*}, \mathbf{1 0}\right]=-\frac{a}{2} \int d^{4} x\left(r_{i j} \chi^{i} \square \chi^{j}+r^{[i j],[l m]} \chi_{[i j]} \square \chi_{[l m]}\right)+\text { h.c. , }
$$

where $r_{i j}\left(r^{[i j],[m]}\right)$ is an arbitrary, nonsingular matrix, symmetrical in $i$ and $j$ (in $[i j]$ and $[l m]$ ). It is then immediately seen that $S_{W}\left[\mathbf{5}^{*}, \mathbf{1 0}\right]$ possess a $\mathbb{Z}_{2}$ symmetry for each of the Weyl left-handed fermion.

Of course, one could wonder if this discrete symmetry is spontaneously broken in the infinite volume limit. In order to fully understand the question of fermion number violation and to clarify in which way the $\mathbb{Z}_{2}$ is realized, one should check that the model has the correct anomaly structure for the left-handed global current. To investigate this important issue, we plan to compute the abelian axial anomaly with Wilson-Majorana fermions.

In the following section we shall study the perturbative behavior of the model. We shall see that it correctly reproduce the continuum perturbation theory properties. 


\section{$5 \quad$ Perturbative Renormalization and One-Loop Calculations}

The consequence of introducing the Wilson-Majorana regularization is that the Slavnov-Taylor identities corresponding to the would-be BRST symmetry do not hold when $a \neq 0$. This allows an a priori large variety of nonsymmetrical contributions to Feynman amplitudes: the question of the possible recovering of the chiral symmetry then arises. The Green functions of the theory defined by $S+S_{\text {c.t. }}$ can satisfy the BRST identities of the formal continuum theory only up to terms vanishing when $a \rightarrow 0$. In this picture, the only purpose of the formal bare theory (the "target theory" of [1]) is to provide us with a number of equations sufficient to determine the coefficients of the monomials in $S_{\text {c.t. }}$. Then it will be possible to perform the final renormalization, i.e. to match the (new) theory $S+S_{c . t .}$ and the continuous one. Equivalently, we could firstly perform a lattice renormalization with the only purpose of fixing the unknown coefficients contained in $S_{\text {c.t. }}$ by requiring that the $a \rightarrow 0$ limit of the Green functions of $S+S_{\text {c.t. }}$ coincides with the continuum result (as a consequence they will also obey the same BRST identities).

The possibility of restoring gauge invariance in a chiral gauge theory adding a suitable collection of nongauge-invariant, non Lorentz-invariant (on the lattice) and also non $U(1)$-invariant (in the Wilson-Majorana version discussed here) terms is suggested by the circumstance that we can consider a noninvariant regularization of QCD (or QED) even though we know how to gauge-invariantly regularize vectorial theories. The difference with the present situation lies in the fact that the gauge-invariant regularization satisfies the Slavnov-Taylor identities, making it possible, through the fine tuning of the free parameters in $S_{\text {c.t. }}$, to obtain the invariant continuum 
theory even starting from the noninvariant regularization, at least in perturbation theory. In a chiral gauge theory the regularized Green functions $G_{\Lambda}\left(x_{1}, \ldots, x_{n}\right)$ are a priori unconstrained, thus casting doubts on the feasibility of this program. We stress that this fine tuning must be performed in a nonperturbative way [41], particularly for the coefficients of the dimensionful couplings. However the perturbative way allows us to perform some simple analytical calculations and checks (at least to lowest order), that can be useful to study renormalizability and to confirm that anomalous dimensions are as in any other continuum regularization.

\subsection{Vertex Function and Propagators}

Here we discuss the structure of the Feynman diagrams we have calculated perturbatively starting from the action functional $S$ defined in (2.11). They are the vacuum polarization tensor, the vertex function and the fermion self-energies (of course we have three different self-energies, as in this theory there are three different propagators for our Weyl fields $\left.\chi^{\alpha}, \bar{\chi}_{\dot{\alpha}}\right)$. We shall then see how these ingredients can be used to fix a number of equations for the unknown coefficients of the interactions contained in $S_{c . t .}$.

\subsubsection{Vacuum Polarization}

We begin by considering the vacuum polarization tensor $\Pi_{\mu \nu}^{a b}(k)$, defined by

$$
\int d^{4} x e^{-i k x}\left\langle A_{\mu}^{a}(x) A_{\nu}^{b}(0)\right\rangle=\frac{\delta_{\mu \nu} \delta^{a b}}{k^{2}}+\frac{\delta_{\mu \rho}}{k^{2}} \Pi_{\rho \sigma}^{a b}(k) \frac{\delta_{\sigma \nu}}{k^{2}}+\cdots
$$

We find that

$$
\begin{aligned}
\Pi_{\mu \nu}^{a b}(k)= & g^{2} \delta_{\mu \nu} \delta^{a b}\left[\delta M_{a}^{2}(r)+k^{2} G_{1}^{a}(r)+k_{\mu}^{2} G_{n . c .}^{a}(r)\right]+ \\
& g^{2} \delta^{a b}\left(k^{2} \delta_{\mu \nu}-k_{\mu} k_{\nu}\right)\left[G_{0} \ln a^{2} k^{2}+G_{F}(r)\right] .
\end{aligned}
$$

\footnotetext{
${ }^{6}$ We work in the Feynman gauge, $\alpha=1$.
} 
Here the $U(1)$ symmetry breaking shows up very clearly: we can not only make fermion-antifermion pairs circulate in loops, but also fermion-fermion as well as antifermion-antifermion pairs, since we do not have to respect fermion number conservation. The consequence on the vacuum polarization tensor is the explicit dependence of the coefficients $\delta M_{a}^{2}(r), G_{1}^{a}(r), G_{n . c .}^{a}(r)$ in (5.2) on the color index $a$. Note that the only logarithmic divergence is the same of the continuum one. Moreover, using dimensional regularization we see that the coefficient of this divergence is again equal to $G_{0}$, where $G_{0}=1 / 48 \pi^{2}$. On the other hand, a mass term $\delta M_{a}^{2}(r) \propto r a^{-2}$ has emerged, which renormalize differently the mass of the gauge fields $A_{\mu}^{a}$ for different values of the color index $a=1,2,3$. Of course, in a vectorial theory, like QCD or QED, regularized in a gauge-invariant way (e.g., on the lattice, by suitably connecting the fermionic matter fields in the Wilson term with the gauge links), a term like this can not appear. In our case, however, gauge invariance (or, with the gauge-fixed action, BRST invariance) is broken by the Wilson-Majorana term, and we can only expect that it has to vanish when $r \rightarrow 0$, as we have verified. The same arguments apply to the coefficient $G_{n . c .}^{a}(r)$ of the $k_{\mu}^{2} \delta_{\mu \nu}$ term, which explicitly breaks Lorentz invariance (still satisfying the unbroken hypercubical lattice symmetry), as well as to $G_{1}^{a}(r)$.

Let us briefly discuss the structure of the tensor $\Pi_{\mu \nu}^{a b}(k)$ in color space. The regularized action does not display the global $S U(2)$ and $U(1)$ symmetries of the continuum theory, and it is not a priori obvious that it should be proportional to $\delta^{a b}$. In fact, this simple structure stems from the three surviving symmetries of the regularized action (that we mentioned in the previous section), which require

$$
\left\langle A_{\mu}^{a}(x) A_{\nu}^{b}(0)\right\rangle=0 \text { if } a \neq b \quad, \quad\left\langle A_{\mu}^{1}(x) A_{\nu}^{1}(0)\right\rangle=\left\langle A_{\mu}^{3}(x) A_{\nu}^{3}(0)\right\rangle
$$


As a consequence, the general form of $\Pi_{\mu \nu}^{a b}(k)$ will be

$$
\Pi_{\mu \nu}^{a b}(k)=\delta^{a b} \Pi_{\mu \nu}^{a}(k), \quad \Pi_{\mu \nu}^{1}(k)=\Pi_{\mu \nu}^{3}(k)
$$

We have also checked that the coefficients in (5.2) satisfy the second condition in $(5.3)$.

\subsubsection{Fermion Self-Energies}

We start with the diagonal propagator of Weyl fields $S_{A B}^{\dot{\alpha} \alpha}(p)$, which we write as

$$
\int d^{4} x e^{-i p x}\left\langle\chi_{A}^{\alpha}(x) \bar{\chi}_{B}^{\dot{\alpha}}(0)\right\rangle=P_{A B}^{\dot{\alpha} \alpha}(p)+P_{A C}^{\dot{\alpha}_{1} \alpha}(p)\left[i \Sigma_{\dot{\alpha}_{1} \beta_{2}}(p)\right]_{C D} P_{D B}^{\dot{\alpha} \beta_{2}}(p)+\cdots,
$$

where

$$
\left[\Sigma_{\dot{\alpha} \beta}\right]_{A B}(p)=g^{2} p_{\dot{\alpha} \beta} \delta_{A B}\left[A \log a^{2} p^{2}+B(r)\right] .
$$

As in (5.2), dimensional regularization gives the same result for the coefficient of the logarithmic pole, $A=C_{F} / 16 \pi^{2}$, where $C_{F}=T_{f}^{b} T_{f}^{b}$. For $S U(2)$, $C_{F}=3 / 4$.

We can observe that the complete propagator is still diagonal in color

indices; this can be explained using the discrete symmetries (3.3), (3.4) of the regularized action, which imply

$$
\left\langle\chi_{A}^{\alpha}(x) \bar{\chi}_{B}^{\dot{\alpha}}(0)\right\rangle \propto \delta_{A B}
$$

In the following, we shall need to extract the $r \rightarrow 0^{+}$part of the Feynman amplitudes, which are automatically BRST invariant, as in [1]: then we write

$$
\Sigma_{\dot{\alpha} \beta}(p)=\left[\Sigma_{\dot{\alpha} \beta}(p)\right]_{r=0^{+}}+g^{2} p_{\dot{\alpha} \beta} G(r),
$$

where now $G(0)=0$. 
As remarked before, the global $U(1)$ symmetry which would ensure fermion number conservation, is broken at the cutoff level by the Wilson-Majorana term, which formally vanishes when $a \rightarrow 0$. Consequently, the zeroth order approximation, $P_{A B}^{\alpha \beta}(p)$, of the nondiagonal propagator, $S_{A B}^{\alpha \beta}(p)$, also vanishes in this limit. However, when inserted in loops, it can give rise to finite as well as to ultraviolet divergent contributions to the Feynman amplitudes: in the present case it produces a Majorana mass renormalization, which for finite $a$ reads

$$
\int d^{4} x e^{-i p x}\left\langle\chi_{A}^{\alpha}(x) \chi_{B}^{\beta}(0)\right\rangle=P_{A B}^{\alpha \beta}(p)+P^{\dot{\alpha}_{1} \alpha}(p)\left[\Sigma_{\dot{\alpha}_{1} \dot{\alpha}_{2}}\right]_{A B} P^{\dot{\alpha}_{2} \beta}(p)+\cdots,
$$

where

$$
\left[\Sigma_{\dot{\alpha} \dot{\beta}}\right]_{A B}=g^{2} \delta_{A B} \epsilon_{\dot{\alpha} \dot{\beta}} \delta m(r),
$$

with $\delta m(r) \propto r a^{-1}$ and, as for the bosonic mass term, $\delta m(0)=0$. When the cutoff is removed, the two diagonal propagators in the second term tend to their continuum limit, $P^{\dot{\alpha} \beta}(p) \rightarrow-i p^{\dot{\alpha} \beta} / p^{2}$, and the result is a linearly divergent term. In passing, we remark that the diagonal color structure of $\left[\Sigma_{\dot{\alpha} \dot{\beta}}\right]_{A B}$ is again a direct consequence of the discrete symmetries of the theory we recorded in the last section.

We omit to write down the obvious formula for the self-energy of antimatter fields, which is obtained from (5.8) by replacing $\epsilon_{\dot{\alpha} \dot{\beta}}$ with $\epsilon_{\beta \alpha}$.

\subsubsection{Vertex Function}

Finally, we present the results of our perturbative calculations for the oneparticle-irreducible (1PI) vertex function $\tilde{\Gamma}_{\dot{\rho} \rho, \mu ; A B}^{a}\left(p^{\prime}, p\right)$. We find that

$$
\begin{aligned}
& \tilde{\Gamma}_{\dot{\rho} \rho, \mu ; A B}^{a}\left(p^{\prime}, p\right)=\left[\tilde{\Gamma}_{\dot{\rho} \rho, \mu ; A B}^{a}\left(p^{\prime}, p\right)\right]_{r=0^{+}}+ \\
& \left(-i g \bar{\sigma}_{\dot{\rho} \rho, \mu}\right)\left[\left(1+g^{2} \gamma_{0} \ln a^{2} \mu^{2}+g^{2} \Gamma_{1}(r)\right) T_{A B}^{a}+g^{2} \Gamma_{2}(r)\left(T^{a}\right)_{A B}^{*}\right]
\end{aligned}
$$


where we have included in the first term the $r \rightarrow 0^{+}$finite corrections to the zeroth order vertex $-i g \bar{\sigma}_{\dot{\rho} \rho, \mu} T_{A B}^{a}$; the functions $\Gamma_{i}(r), i=1,2$ are both independent of $p^{\prime}, p$, and satisfy the condition $\Gamma_{i}(0)=0$.

Let us note, in the previous formula, the appearance of the generators of the color symmetry in the antifundamental representation, $\left(T^{a}\right)_{A B}^{*}$ : this is due to the insertion of nondiagonal Weyl propagators in virtual loops, as an effect of the breaking of global $S U(2)$ and $U(1)$ symmetries in the regularized theory. Lastly, we have checked that the coefficient of the logarithmic divergence is again the same as in the continuum, i.e. $\gamma_{0}=\left(C_{F}+C_{a d j}\right) / 16 \pi^{2}, C_{a d j}$ being the Dynkin index of the adjoint representation. For $S U(2), C_{a d j}=2$.

\subsection{Slavnov-Taylor Identities}

Following the ideas sketched in the last section, we now examine a number of Slavnov-Taylor identities to determine the structure of $S_{\text {c.t. }}$. We start by considering the gauge field sector. The relevant identity is]

$$
\left\langle\delta\left(\bar{c}^{a}(x) A_{\nu}^{b}(y)\right)\right\rangle=0
$$

which, using (3.1), becomes

$$
\frac{1}{\alpha}\left\langle\partial_{\mu}^{x} A_{\mu}^{a}(x) A_{\nu}^{b}(y)\right\rangle+\left\langle\bar{c}^{a}(x)\left(D_{\nu} c\right)^{b}(y)\right\rangle=0 .
$$

Differentiating with respect to $y_{\nu}$ and using the equations of motion for ghost fields, we get

$$
\partial_{\mu}^{x} \partial_{\nu}^{y}\left\langle A_{\mu}^{a}(x) A_{\nu}^{b}(y)\right\rangle=\alpha \delta^{(4)}(x-y)
$$

which shows that the longitudinal part of the gauge field propagator is unrenormalized by the interaction. This identity is automatically satisfied by the pure gauge and ghost contributions to the vacuum polarization tensor

\footnotetext{
${ }^{7}$ We use continuum notation, for the sake of simplicity.
} 
even for $a \neq 0$ (if we gauge-invariantly regularize this sector), but not by terms arising from fermion matter fields. By adding to the bare action $S$ the appropriate counterterms of dimension less than or equal to four we called $S_{c . t .}$, we will have

$$
\Pi_{\mu \nu}^{a b}(k) \longrightarrow \Pi_{\mu \nu}^{a b}(k)+\left[\Pi_{\mu \nu}^{a b}\right]_{c . t .}(k) .
$$

Is it now possible to satisfy the identity (5.10) up to terms of $\mathrm{O}(a)$ ? By Fourier transforming into momentum space we obtain the following equation

$$
k_{\mu} k_{\nu}\left(\Pi_{\mu \nu}^{a b}(k)+\left[\Pi_{\mu \nu}^{a b}\right]_{c . t .}(k)\right)=0,
$$

which fixes a number of coefficients for the interactions in $S_{c . t .}$. In fact, (5.13) requires

$$
\left[\Pi_{\mu \nu}^{a b}\right]_{c . t .}(k)=-g^{2} \delta^{a b}\left[\delta_{\mu \nu} \delta M_{a}^{2}(r)+k_{\mu}^{2} \delta_{\mu \nu} G_{n . c .}^{a}(r)+k_{\mu} k_{\nu} G_{1}^{a}(r)\right] .
$$

This means that we have to add to the bare action a gauge- and Lorentz-variant (but still renormalizable) counterterm of the form

$$
\frac{1}{2} \int d^{4} x \sum_{a}\left(\delta M_{a}^{2} A_{\mu}^{a} A_{\mu}^{a}+G_{n . c .}^{a}(r) \sum_{\mu}\left(\partial_{\mu} A_{\mu}^{a}\right)\left(\partial_{\mu} A_{\mu}^{a}\right)+G_{1}^{a}(r)\left(\partial_{\mu} A_{\mu}^{a}\right)^{2}(x)\right) .
$$

In the fermion sector, we have to impose the following three Slavnov-Taylor identities to force the regularized theory to reproduce the continuum theory BRST symmetry:

$$
\begin{gathered}
\left\langle\delta\left(\chi_{A}^{\alpha}(x) \bar{\chi}_{B}^{\dot{\beta}}(y) \bar{c}^{a}(z)\right)\right\rangle=0 \\
\left\langle\delta\left(\chi_{A}^{\alpha}(x) \chi_{B}^{\beta}(y) \bar{c}^{a}(z)\right)\right\rangle=0, \quad\left\langle\delta\left(\bar{\chi}_{A}^{\dot{\alpha}}(x) \bar{\chi}_{B}^{\dot{\beta}}(y) \bar{c}^{a}(z)\right)\right\rangle=0
\end{gathered}
$$

that is

$$
\begin{aligned}
& \frac{1}{\alpha}\left\langle\partial_{\mu}^{z} A_{\mu}^{a}(z) \chi_{A}^{\alpha}(x) \bar{\chi}_{B}^{\dot{\beta}}(y)\right\rangle-i g\left\langle\bar{c}^{a}(z) c^{b}(x)\left(T^{b} \chi^{\alpha}(x)\right)_{A} \bar{\chi}_{B}^{\dot{\beta}}(y)\right\rangle \\
& +i g\left\langle\bar{c}^{a}(z) \chi_{A}^{\alpha}(x)\left(\bar{\chi}^{\dot{\beta}}(y) T^{b}\right)_{B} c^{b}(y)\right\rangle=0,
\end{aligned}
$$




$$
\begin{aligned}
& \frac{1}{\alpha}\left\langle\partial_{\mu}^{z} A_{\mu}^{a}(z) \chi_{A}^{\alpha}(x) \chi_{B}^{\beta}(y)\right\rangle-i g\left\langle\bar{c}^{a}(z) c^{b}(x)\left(T^{b} \chi^{\alpha}(x)\right)_{A} \chi_{B}^{\beta}(y)\right\rangle \\
& +i g\left\langle\bar{c}^{a}(z) \chi_{A}^{\alpha}(x) c^{b}(y)\left(T^{b} \chi^{\beta}(y)\right)_{B}\right\rangle=0 \\
& \frac{1}{\alpha}\left\langle\partial_{\mu}^{z} A_{\mu}^{a}(z) \bar{\chi}_{A}^{\dot{\alpha}}(x) \bar{\chi}_{B}^{\dot{\beta}}(y)\right\rangle+i g\left\langle\bar{c}^{a}(z) c^{b}(x)\left(\bar{\chi}^{\dot{\alpha}}(x) T^{b}\right)_{A} \bar{\chi}_{B}^{\dot{\beta}}(y)\right\rangle \\
& -i g\left\langle\bar{c}^{a}(z) \bar{\chi}_{A}^{\dot{\alpha}}(x) c^{b}(y)\left(\bar{\chi}^{\dot{\beta}}(y) T^{b}\right)_{B}\right\rangle=0 .
\end{aligned}
$$

We start by examining the first identity. Writing the fermion-fermion-gauge Green function in terms of 1PI vertex function, complete fermion propagators and complete gauge field propagator, whose longitudinal part is fixed by means of (5.11), we get

$$
\begin{aligned}
& \frac{1}{\alpha} \int d^{4} x d^{4} y e^{i q x+i p y}\left\langle\partial_{\mu}^{x} A_{\mu}^{a}(x) \chi_{A}^{\alpha}(0) \bar{\chi}_{B}^{\dot{\beta}}(y)\right\rangle= \\
& -i \frac{q_{\nu}}{q^{2}} S^{\dot{\rho} \alpha}(q+p) \tilde{\Gamma}_{\dot{\rho} \rho, \nu ; A B}^{a}(q+p, p) S^{\dot{\beta} \rho}(p) .
\end{aligned}
$$

The second and the third term of the identity (5.18) are seen to be, for $a \neq 0$,

$$
\begin{gathered}
\sum_{x, y} a^{8} e^{i q x+i p y}\left\langle\bar{c}^{a}(x) c^{b}(0)\left(T^{b} \chi^{\alpha}(0)\right)_{A} \bar{\chi}_{B}^{\dot{\beta}}(y)\right\rangle= \\
-\triangle_{g h}(q) T_{A B}^{a}\left[S^{\dot{\beta} \alpha}(p)+\bar{\sigma}_{\tau}^{\dot{\rho} \alpha} \bar{\sigma}_{\dot{\rho} \rho, \lambda} S^{\dot{\beta} \rho}(p) g^{2} K_{\tau \lambda}(p, q)\right] \\
\sum_{x, y} a^{8} e^{i q x+i p y}\left\langle\bar{c}^{a}(x) \chi_{A}^{\alpha}(0)\left(\bar{\chi}^{\dot{\beta}}(y) T^{b}\right)_{B} c^{b}(y)\right\rangle= \\
-\triangle_{g h}(q) T_{A B}^{a}\left[S^{\dot{\beta} \alpha}(p+q)+\right. \\
\left.S^{\dot{\rho} \alpha}(p+q) \bar{\sigma}_{\dot{\rho} \rho, \lambda} \bar{\sigma}_{\tau}^{\dot{\beta} \rho} g^{2} K_{\tau \lambda}(-p-q, q)\right]
\end{gathered}
$$

where $\triangle_{g h}(q)$ is the ghost propagator. $K_{\tau \lambda}(p, q)$ is a complicated function of the external momenta $p, q$ and of the Wilson parameter $r$; its complete expression can be found in Appendix B. The crucial point is that, extracting the $r \rightarrow 0^{+}$part, we obtain

$$
K_{\tau \lambda}(p, q)=\left[K_{\tau \lambda}(p, q)\right]_{r=0^{+}}+\delta_{\tau \lambda} K(r),
$$


where now $K(r)$ only depends on $r$. Substituting these expressions into (5.18), the $r=0^{+}$terms in (5.24) satisfy this identity, whereas the $r$-dependent ones do not. We are thus lead to introduce a new interaction in the bare action in order to compensate for these terms, i.e.

$$
Z_{2} \int d^{4} x \bar{\chi}_{A}^{\dot{\alpha}} \partial_{\dot{\alpha} \beta, \mu} \chi_{A}^{\beta}+Z_{1} \int d^{4} x \bar{\chi}_{A}^{\dot{\alpha}}\left(i g A_{\dot{\alpha} \beta}\right)_{A B} \chi_{B}^{\beta}+\tilde{Z}_{1} \int d^{4} x \bar{\chi}_{A}^{\dot{\alpha}}\left(i g A_{\dot{\alpha} \beta}\right)_{B A} \chi_{B}^{\beta} .
$$

Imposing (5.18), we get the following equations:

$$
Z_{1}-Z_{2}=-g^{2}\left(G+\Gamma_{1}+K\right)(r) \quad, \quad \tilde{Z}_{1}=-g^{2} \Gamma_{2}(r)
$$

It will not be possible (and actually it is not necessary) to fix separately the two constants $Z_{1}, Z_{2}$. In fact, we are only demanding that, for a generic string of fields $O\left(x_{1}, \ldots, x_{n}\right)$, the identities

$$
\left\langle\delta O\left(x_{1}, \ldots, x_{n}\right)\right\rangle=\left\langle O\left(x_{1}, \ldots, x_{n}\right) \delta\left(S_{W}+S_{c . t .}\right)\right\rangle=0
$$

should be satisfied up to terms vanishing when $a \rightarrow 0$. When the counterterm (5.25) is inserted in the previous equation, only the difference $Z_{1}-Z_{2}$ can appear, since

$$
\delta\left(\bar{\chi}_{A}^{\dot{\alpha}}\left(\partial_{\dot{\alpha} \beta}+i g A_{\dot{\alpha} \beta}\right)_{A B} \chi_{B}^{\beta}\right)=0
$$

Of course, when the final renormalization is performed, all the coefficients of the counterterms will acquire their well-definite values, once appropriate renormalization conditions are imposed.

Finally, we briefly examine identities (5.19) and (5.20). In the one loop approximation, non BRST-invariant contributions can only arise from first order corrections to the nondiagonal propagators for fermion fields, which are responsible for a Majorana mass renormalization. The introduction of a counterterm of the form

$$
\frac{M}{2} \int d^{4} x\left(\bar{\chi}^{\dot{\alpha}} \bar{\chi}^{\dot{\beta}} \epsilon_{\dot{\alpha} \dot{\beta}}+\chi^{\alpha} \chi^{\beta} \epsilon_{\beta \alpha}\right)
$$


will make the corresponding identity satisfied, provided that

$$
M=\delta m(r)
$$

The same equation could have also been obtained by noting that a mass term is not even invariant with respect to global $S U(2)$ or $U(1)$ transformations. One of the consequences of this observation is that we have, for the two point function $\left\langle\chi_{A}^{\alpha}(x) \chi_{B}^{\beta}(0)\right\rangle$,

$$
T_{A C}^{a}\left\langle\chi_{C}^{\alpha}(x) \chi_{B}^{\beta}(0)\right\rangle+T_{B C}^{a}\left\langle\chi_{A}^{\alpha}(x) \chi_{C}^{\beta}(0)\right\rangle=0
$$

that is, for $A=B$,

$$
T_{A C}^{a}\left(\left\langle\chi_{C}^{\alpha}(x) \chi_{A}^{\beta}(0)\right\rangle-\left\langle\chi_{C}^{\beta}(0) \chi_{A}^{\alpha}(x)\right\rangle\right)=0
$$

This condition together with a similar one for antimatter fields is trivially satisfied just when the counterterm in (5.28) is included in the action functional of the regularized theory.

\section{Conclusions}

We have presented here a few one-loop calculations in a simple anomaly-free model of lattice chiral gauge theory. They lend strong support to the idea that it is possible to reach the BRST-invariant, continuum theory, starting from an action functional which is the sum of a Wilson regularization of the tree level action plus a finite number of renormalizable counterterms. In the one-loop approximation, they are given by (5.15), (5.25), (5.28). The coefficients of these interactions must be fine-tuned in order to match the continuum target theory onto its regularized form. Our regularization makes use of the physical, left-handed degrees of freedom of the theory only, and in perturbation theory we found no obstructions which would prevent this 
program to be carried out successfully. The correctness of this procedure at the nonperturbative level cannot be settled at this stage of work, and should be assumed in any forthcoming Monte Carlo simulation. As discussed in section 4, a study of nonperturbative (instanton) physics could clarify the issue of the existence of a $\mathbb{Z}_{2}$ simmetry in the regularized theory. Since the model correctly reproduce perturbation theory, we do not expect essential problems. We plan to come back on this point in a future publication.

\section{Acknowledgments}

It is a great pleasure to thank my thesis advisor, Massimo Testa, for suggesting me this problem, for his constant encouragement and support, and for many interesting discussions. I would also like to thank Francesco Fucito and Gian Carlo Rossi for a critical reading of this manuscript, and the Referee for comments and discussions on the issue of fermion number nonconservation. 


\section{A Euclidean $\bar{\sigma}$ Matrices}

It is well known that there is a one-to-one correspondence between Weyl spinors with one dotted and one undotted index (four independent components) and ordinary 4-vectors [24], which can be written as

$$
p_{\dot{\alpha} \beta}=\sum_{\lambda=1}^{4} p_{\lambda} \bar{\sigma}_{\dot{\alpha} \beta, \lambda},
$$

where the four $2 \times 2$ independent matrices $\bar{\sigma}_{\lambda}$ are defined through the anticommutation relations they must obey, i.e.

$$
\begin{aligned}
& \bar{\sigma}_{\dot{\alpha} \beta, \mu} \bar{\sigma}_{\nu}^{\dot{\rho} \beta}+\bar{\sigma}_{\dot{\alpha} \beta, \nu} \bar{\sigma}_{\mu}^{\dot{\rho} \beta}=2 \delta_{\mu \nu} \epsilon_{\dot{\alpha}}^{\dot{\rho}}, \\
& \bar{\sigma}_{\dot{\alpha} \beta, \mu} \bar{\sigma}_{\nu}^{\dot{\alpha} \rho}+\bar{\sigma}_{\dot{\alpha} \beta, \nu} \bar{\sigma}_{\mu}^{\dot{\alpha} \rho}=2 \delta_{\mu \nu} \epsilon_{\beta}^{\rho} .
\end{aligned}
$$

Starting from (A.2), (A.3), it is possible to derive some very useful relations for products (and traces of products) of $\bar{\sigma}$ matrices. We collect here some of these properties in $D$ dimensions.

$$
\begin{gathered}
\sum_{\mu} \bar{\sigma}_{\mu}^{\dot{\beta} \alpha} \bar{\sigma}_{\dot{\rho} \alpha, \mu}=D \epsilon_{\dot{\rho}}^{\dot{\beta}} \\
\sum_{\mu} \bar{\sigma}_{\dot{\beta} \alpha, \mu} \bar{\sigma}_{\rho}^{\dot{\gamma} \alpha} \bar{\sigma}_{\dot{\gamma} \delta, \mu}=(-D+2) \bar{\sigma}_{\dot{\beta} \delta, \rho} \\
\sum_{\mu} \bar{\sigma}_{\dot{\beta} \alpha, \mu} \bar{\sigma}_{\rho_{1}}^{\dot{\gamma} \alpha} \bar{\sigma}_{\dot{\gamma} \delta, \rho_{2}} \bar{\sigma}_{\mu}^{\dot{\theta} \delta}=4 \epsilon_{\rho_{1} \rho_{2}} \epsilon_{\dot{\beta}}^{\dot{\theta}}+(D-4) \bar{\sigma}_{\dot{\beta} \delta, \rho_{1}} \bar{\sigma}_{\rho_{2}}^{\dot{\theta} \delta} \\
\sum_{\mu} \bar{\sigma}_{\dot{\beta} \alpha, \mu} \bar{\sigma}_{\rho_{1}}^{\dot{\gamma} \alpha} \bar{\sigma}_{\dot{\gamma} \delta, \rho_{2}} \bar{\sigma}_{\rho_{3}}^{\dot{\eta} \delta} \bar{\sigma}_{\dot{\eta} \theta, \mu}=-2 \bar{\sigma}_{\dot{\beta} \alpha, \rho_{3}} \bar{\sigma}_{\rho_{2}}^{\dot{\gamma} \alpha} \bar{\sigma}_{\dot{\gamma} \theta, \rho_{1}}-(D-4) \bar{\sigma}_{\dot{\beta} \delta, \rho_{1}} \bar{\sigma}_{\rho_{2}} \dot{\theta} \delta \bar{\sigma}_{\dot{\eta} \theta, \rho_{3}} \\
\bar{\sigma}_{\mu}^{\dot{\beta} \alpha} \bar{\sigma}_{\dot{\beta} \alpha, \nu}=f(D) \delta_{\mu \nu},
\end{gathered}
$$

where

$$
f(D)=\operatorname{Tr} \mathbb{1}
$$

hence $f(4)=2$. 


\section{B The Explicit Expression of the Function $K_{\mu \nu}(p, q)$}

Here we report the result of our perturbative calculations for the function

$K_{\mu \nu}(p, q)$ which appears in the Slavnov-Taylor identities (5.22), (5.23). It is readily evaluated to be

$$
K_{\mu \nu}(p, q)=\frac{C_{a d j}}{2} \cos \frac{q_{\nu} a}{2} \int \frac{d^{4} k}{(2 \pi)^{4}} \frac{2 \sin k_{\mu} \sum_{\lambda} \sin \left(\frac{k+(p+q) a}{2}\right)_{\lambda} \cos \left(\frac{k-p a}{2}\right)_{\lambda} \delta_{\lambda \nu}}{\Delta(k) \tilde{\Delta}(k+p a) \tilde{\Delta}(k+p a+q a)}
$$

where

$$
\tilde{\Delta}(k)=\frac{1}{4 \sum_{\mu} \sin ^{2} \frac{k_{\mu}}{2}},
$$

and the function $\Delta(k)$ is defined in (2.8). It should be pointed out that the only dependence on the Wilson parameter is contained in the function $\tilde{\Delta}$. According to the decomposition (5.24), we then obtain from (B.1), evaluated in the limit $a \rightarrow 0$, the result

$$
K(r)=-\frac{C_{a d j}}{8} \int \frac{d^{4} k}{(2 \pi)^{4}} \frac{1}{[\tilde{\Delta}(k)]^{2}}\left(\frac{D(k)}{\Delta(k)}-1\right),
$$

from which it follows that $K(r) \rightarrow 0$ as $r \rightarrow 0^{+}$. 


\section{References}

[1] A. Borrelli, L. Maiani, G. C. Rossi, R. Sisto and M. Testa, Nucl. Phys. B333 (1990) 335.

[2] D. Kaplan, Phys. Lett. B288 (1992) 342; Nucl. Phys. B (Proc. Suppl.) 30 (1993) 597.

[3] L. Maiani, G. C. Rossi and M. Testa, Phys. Lett. B292 (1992) 397.

[4] R. Narayanan and H. Neuberger, Phys. Lett. B302 (1993) 62; Nucl. Phys. B412 (1994) 574; ibid. B443 (1995) 305.

[5] S. A. Frolov and A. A. Slavnov, Nucl. Phys. B411 (1994) 647.

[6] G. 't Hooft, Phys. Lett. B349 (1995) 491.

[7] P. Hernandez and R. Sundrum, Nucl. Phys. B455 (1995) 287; ibid. B472 (1996) 334.

[8] J. Alonso, Ph. Boucaud, F. Lesmes and A. J. van der Sijs, Nucl. Phys. B457 (1995) 175; ERRATUM, ibid. B472 (1996) 738.

[9] W. Bietenholz and U.-J. Wiese, Phys. Lett. B378 (1996) 222.

[10] S. D. H. Hsu, Regularization of Chiral Gauge Theories, preprint YCTPP5-9, hep-lat/9503064.

[11] G. T. Bodwin, A Lattice Formulation of Chiral Gauge Theories, preprint ANL-HEP-PR-95-59-REV, hep-lat/9510002.

[12] Y. Shamir, The Standard Model From a New Phase Transition on the Lattice, preprint TAUP-2306-95, hep-lat/9512019; Lattice Chiral Gauge Theories in a Renormalizable Gauge, preprint TAUP-2362-96, hep-lat/9608117. 
[13] M. F. L. Golterman and Y. Shamir, A Gauge-Fixing Action for Lattice Gauge Theories, preprint TAUP-2361-96, UTCCP-P-11, Wash. U. HEP/96-60, hep-lat/9608116.

[14] M. Testa, The Rome Approach to Chirality, talk presented at the APCTP-ICTP Joint International Conference 1997, hep-lat/9707007.

[15] S. Adler, Phys. Rev. 177 (1969) 2426.

[16] J. Bell and R. Jackiw, Nuovo Cimento 60A (1969) 47.

[17] See e.g. C. Itzykson and J. B. Zuber, Quantum Field Theory, Mc GrawHill International Editions, 1985.

[18] Y. Meurice and G. Veneziano, Phys. Lett. 141B (1984) 69.

[19] I. A. Affleck, M. Dine and N. Seiberg, Phys. Rev. Lett. 53 (1984) 1677.

[20] D. Amati, K. Konishi, Y. Meurice, G.C. Rossi and G. Veneziano, Phys. Rep. 162 (1988) 169, and references therein.

[21] D. Gross and R. Jackiw, Phys. Rev. D6 (1972) 477.

[22] L. Maiani, Nucl. Phys. B (Proc. Suppl.) 29B,C (1992) 33.

[23] M. Testa, Nucl. Phys. B (Proc. Suppl.) XXX (1993) 1.

[24] B. L. van der Waerden, Group Theory and Quantum Mechanics, Springer-Verlag, 1974.

[25] E. Witten, Phys. Lett. B117 (1982) 324.

[26] K. G. Wilson, Phys. Rev. D10 (1974) 2445.

[27] G. C. Rossi, R. Sarno and R. Sisto, Nucl. Phys. B398 (1993) 101. 
[28] R. Sarno and R. Sisto, Nucl. Phys. B (Proc. Suppl.) 29B,C (1992) 152.

[29] C. Becchi, A. Rouet and R. Stora, Phys. Lett. B52 (1974) 344.

[30] I. V. Tyutin, Gauge invariance in field theory and in statistical physics in the operator formulation, Lebedev preprint FIAN No. 39 (1975), unpublished.

[31] H. B. Nielsen and M. Ninomiya, Nucl. Phys. B185 (1981) 20.

[32] H. B. Nielsen and M. Ninomiya, Nucl. Phys. B193 (1981) 173.

[33] G. 't Hooft, Phys. Rev. Lett. 37 (1976) 8.

[34] G. 't Hooft, Phys. Rev D14 (1976) 3432; ibid. D18 (1978) 2199.

[35] A. S. Schwarz, Phys. Lett. 67 (1977) 172; Comm. Math. Phys. 64 (1979) 233.

[36] R. Jackiw and C. Rebbi, Phys. Rev. D16 (1977) 1052.

[37] N. Nielsen and B. Schroer, Nucl. Phys. B127 (1977) 493.

[38] S. Coleman, The Uses of Instantons, Lecture delivered at 1977 International School of Subnuclear Physics, Erice, Italy.

[39] R. Narayanan and P. Vranas, A numerical test of the continuum index theorem on the lattice, hep-lat/9702005, and references therein.

[40] H. Neuberger, A Lecture on Chiral Fermions, talk given at the DESY workshop, September 1995, hep-lat/9511001.

[41] G. Parisi, Nucl. Phys. B (Proc. Suppl.) 29B,C (1992) 247. 